\title{
On the use of $\alpha$-shapes for the measurement of 3D bubbles in fluidized beds from Two-Fluid Model simulations
}

\author{
Ignacio Julián ${ }^{1}$, David González ${ }^{2}$, Javier Herguido ${ }^{1}$, Miguel Menéndez ${ }^{1}$ \\ ${ }^{1}$ Catálisis, separaciones moleculares e ingeniería de reactores (CREG) \\ ${ }^{2}$ Mecánica aplicada y bioingeniería (AMB) \\ Instituto de Investigación en Ingeniería de Aragón (I3A) \\ Universidad de Zaragoza, Mariano Esquillor s/n, 50018, Zaragoza, Spain. \\ Tel.+34-976762707, e-mail: ijulian@unizar.es
}

\begin{abstract}
A geometrical technique based on shape construction was employed to reconstruct the simulated domain of 3D bubbles in gas-solid fluidized beds from Two-Fluid Model simulations. The Delaunay triangulation of the cloud of points that represent volume fraction iso-surfaces was filtered using $\alpha$-shapes, allowing a topologically accurate description of the bubbles.
\end{abstract}

\section{Introduction}

The analysis of bubble characteristics from TwoFluid Model (TFM) simulations of 3D gas-solid fluidized beds is not straightforward. The commercial Computational Fluid Dynamic (CFD) codes are able to post-process transient results to show bubble contours by means of void fraction isosurfaces but they are not able to discriminate single gas bubbles. Therefore, bubble data cannot be directly extracted from the simulation results.

Some authors [1] use tomography techniques to get radial voidage maps at different bed heights and then carry out image reconstruction to estimate 3D bubble characteristics. Some others [2] couple porosity maps and bubble shape factors from single detached bubble simulations to estimate 3D bubble characteristics. In any case, the 3D bubble volume estimation from 2D planar porosity maps is computationally expensive and the accuracy of the method depends on the number of sliced planes considered in the 3D image reconstruction [1].

Taking advantage of the characteristic fixed mesh which is used in the TFM formulation for the simulation of the fluidized bed dynamics, this work suggest a direct method to quantify 3D bubble properties from nodal results applying a geometric construction technique, the so-called $\alpha$-shape method introduced by Edelsbrunner and Mücke [3]. The use of $\alpha$-shapes allows a topological reconstruction of individual bubbles creating unconnected subdomains whose contours are the individual bubble boundaries. The accuracy of the bubble contour reconstruction depends on the computational mesh size, i.e. the distance between adjacent mesh nodes. The geometric reconstruction based on $\alpha$-shapes, which is extensively applied in other fields such medical image analysis or molecular structure modeling, is here used for the first time to identify and measure gas bubbles in simulated 3D fluidized beds.

This study specifically focuses on the detection and measurement of 3D bubbles in a particular fluidized bed reactor configuration: the Two-Section TwoZone Fluidized Bed Reactor (TS-TZFBR). This reactor has been described elsewhere as a potential tool for process intensification in the field of heterogeneous catalysis due to its ability to perform heterogeneous catalytic reactions (e.g. alkane dehydrogenation, ethanol steam reforming or methane aromatization) and catalyst regeneration in a single vessel [4].

The comparison between the TFM bubbling predictions for planar and 3D bed configurations aims to bring some light on the extrapolation of experimental pseudo-2D bubble data to the real bubbling behaviour in a 3D column.

\section{Simulations}

3D-TFM simulations were performed using the commercial software Ansys CFX 14.5. The mass and momentum conservation equations of the TFM model, that considers both solid and gas phase as interpenetrating continua, were solved using finite volumes via spatial and temporal discretization of pressure and velocity fields as well as of transient transport terms. The set of closure equations includes the Gidaspow drag function for the interphase drag force, the model of Lun et al. for the viscous stress and the kinetic theory of granular flow for the granular temperature. A bulk mass flow rate was selected as the boundary condition for the 
reactor gas inlets to avoid dense phase outflow. An opening boundary was applied to the reactor outlet. The flow direction through inlets and outlets was defined as normal to the boundary surface and the relative pressure on the domain outlets was set to $\mathrm{P}_{\mathrm{atm}}$. A no-slip condition was applied for both the gas and solid phases at the walls.

\section{Bubbles discrimination}

In order to identify bubble boundaries, a prescribed solids volume fraction $\varepsilon_{\mathrm{s}}=0.15$ was defined as a threshold value for the iso-surfaces method The domain defined by the iso-surfaces enclosed, then, a list of spatial coordinates of the mesh nodes that accomplish the given restriction.

A Delaunay triangulation algorithm was applied (Matlab R2010a) to connect every node by means of unique tetrahedral shapes. Since the algorithm itself did not discriminate nodes that belong to different bubbles, it was necessary to constrain node connections in order to identify 'false' connections and remove them, generating multiple domains: one for each bubble. The $\alpha$-shapes were then applied to eliminate all tetrahedra whose circumscribing mesh size was greater than a prescribed level of detail for the geometry, $\alpha$.

\section{Results}

3D bubble data from TFM simulations of TSTZFBR were compared to these obtained for pseudo-2D beds. Several formulations were used to describe the equivalent bubble diameter $\left(\mathrm{d}_{\mathrm{b}}\right)$ in order to establish a fair comparison between pseudo-2D and 3D bubble sizes. Volume-based and area-based equivalent diameters were defined accounting for the gas volume enclosed by bubbles (Figures 1.a and 1.b) and the projected bubble area in the axial plane at the bed center (Figures 1.c and 1.d), respectively. The axial evolution of the average bubble diameter for both 3D and pseudo-2D TSTZFBR followed a similar trend, as shown in Figure 2.

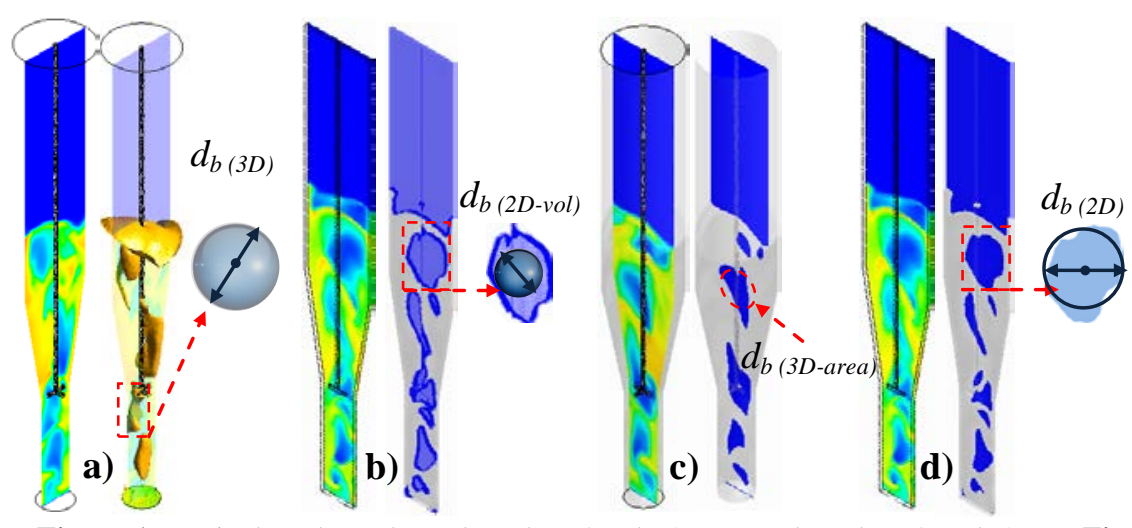

Figure 1. Equivalent $d_{b}$. Volume-based: a) 3D, b) 2D. Area-based: c) 3D, d) 2D Figure 2. Volume-based equivalent $d_{b}(z)$ profiles

\section{Conclusions}

An $\alpha$-shapes method was proposed to measure 3D bubbles from TFM simulations. Compared to the tomographic techniques used in the open literature, this method provided a more reliable tool to analyze the bubble hydrodynamics in simulated 3D fluidized beds.

A volume-based formulation of the equivalent $d_{b}$ for both pseudo-2D and 3D beds gave the most comparable results between reactor configurations. Nevertheless, the average $d_{b, p s e u d o-2 D}$ underestimated $\mathrm{d}_{\mathrm{b}, 3 \mathrm{D}}$ by $23.2 \%$ within the tapered bed region. This reveals that the bed geometry effectively plays a role in the fluid dynamic behaviour of the TSTZFBR and, thus, the extrapolation of experimental fluid dynamic data from pseudo-2D to 3D beds must be done with great care.

\section{REFERENCES}

[1]. SOBRINO,C., ACOSTA-IBORRA, A., IZQUIERDO -BARRIENTOS, M.A., DE VEGA, M. Threedimensional two-fluid modeling of a cylindrical fluidized bed and validation of the maximum entropy method to determine bubble properties. Chemical Engineering Journal. 2015, 262, 628-639.

[2]. VERMA, V., DEEN, N.G., PADDING, J.T., KUIPERS, J.A.M. Two-fluid modeling of threedimensional cylindrical gas-solid fluidized beds using the kinetic theory of granular flow. Chemical Engineering Science. 2013, 102, 227-245.

[3]. EDELSBRUNNER, H., MUCKE, E.P. 3-dimensional alpha-shapes. ACM Transactions on Graphics. 1994, 13(1), 43-72.

[4]. HERGUIDO, J., MENÉNDEZ, M., SANTAMARÍA, $\mathrm{J}$. On the use of fluidized bed catalytic reactors where reduction and oxidation zones are present simultaneously. Catalysis Today. 2005, 100(1-2), 181-189.

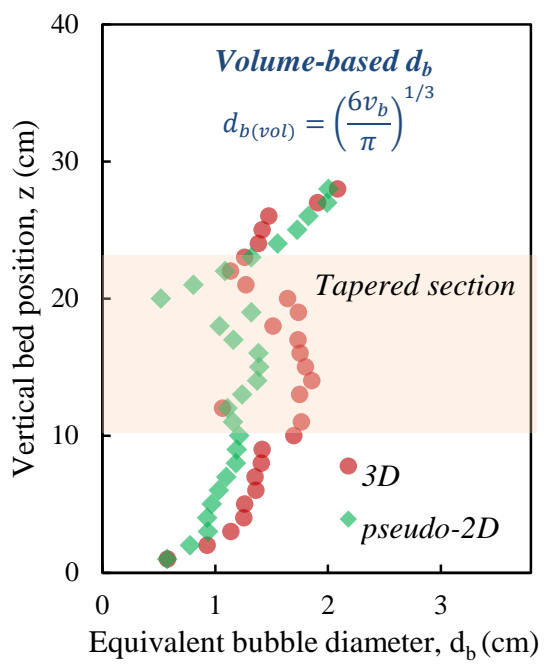

